PERSPECTIVES ON EXPEDITED FDA APPROVAL 685

\title{
Few new drugs deserve expedited regulatory treatment
}

\author{
Jonathan J Darrow, SJD, LLM, JD, MBA
}

Safety testing of new drugs has been required since the 1938 Federal Food, Drug, and Cosmetic Act, but applications were automatically approved under that law unless the US Food and Drug Administration (FDA) acted to prevent marketing within 60 days. In 1962, the Kefauver-Harris Drug Amendments raised the bar by prohibiting marketing until affirmative FDA approval based on "adequate test," showing safety, and "adequate and well-controlled investigations," providing "substantial evidence" of efficacy. The heightened requirements were intended to screen useless or harmful remedies from the market, but they also increased development costs and lengthened the time before drugs were available to patients.

Over the next 6 decades, these increased requirements met resistance, causing the pendulum to swing back, with Congress and the FDA establishing a growing array of programs that reduced evidence requirements and were intended to lower development costs and expedite availability. Some suggest that these more flexible requirements reflect regulatory capture, pointing to industry funding of the FDA, which has grown dramatically since its inception in 1992 and now provides a majority of FDA drug review budgets. ${ }^{1}$ Supporters of expedited programs counter that patients with serious illnesses cannot wait until higher quality evidence is available and that more limited evidence can sometimes adequately satisfy statutory standards.

Regardless of the amount of evidence that should be required for approval, the debate over evidence has often distracted attention from the more fundamental issue of efficacy. Evidence standards relate to certainty, rather than magnitude, of benefit. Even under the heightenend 1962 evidence requirements, there was never any minimum quantum of efficacy that drugs had to possess to receive FDA approval (other than nonzero), ${ }^{2}$ and reviews consistently find that most (69\%-98\%) new drugs fail to provide large benefits over existing therapies (Table 1).

As the new expedited programs for high-priority medicines were implemented, the extent to which such drugs benefitted patients continued to receive little serious attention. For example, the House Report to the 1983 Orphan Drug Act rationalized the use of smaller clinical trial sizes by observing that "dramatically effective" drugs "do not need large patient populations to demonstrate the point," 3 but dramatic effect size was not included as an Orphan Drug Act requirement. The FDA's fast-track regulations, promulgated in 1988 amid the worsening AIDS epidemic, explained that the more limited evidence required under this program was appropriate because "desperately

\author{
Author affiliations \\ Jonathan J Darrow, SJD, LLM, JD, \\ MBA, Program on Regulation, \\ Therapeutics, and Law (PORTAL), \\ Division of Pharmacoepidemiology and \\ Pharmacoeconomics, Department of \\ Medicine, Brigham and Women's Hospital \\ and Harvard Medical School, Boston, MA. \\ AUTHOR CORRESPONDENCE: \\ Jonathan J Darrow, 347.792.2246; \\ jjdarrow@bwh.harvard.edu \\ J Manag Care Spec Pharm. \\ 2021;27(5):685-88 \\ Copyright $@ 2021$, Academy of Managed \\ Care Pharmacy. All rights reserved.
}

ill patients...are generally willing to accept greater risks," but the regulations did not describe any heightened threshold of benefit needed to justify those greater risks beyond what was needed for ordinary approval. ${ }^{4}$

More recent expedited development programs have acknowledged the importance of efficacy while simultaneously establishing flexible criteria that do not require drugs to be more effective than under the traditional de minimis standard, thereby contributing to a growing chasm between public perception of what FDA approval means and the degree of 


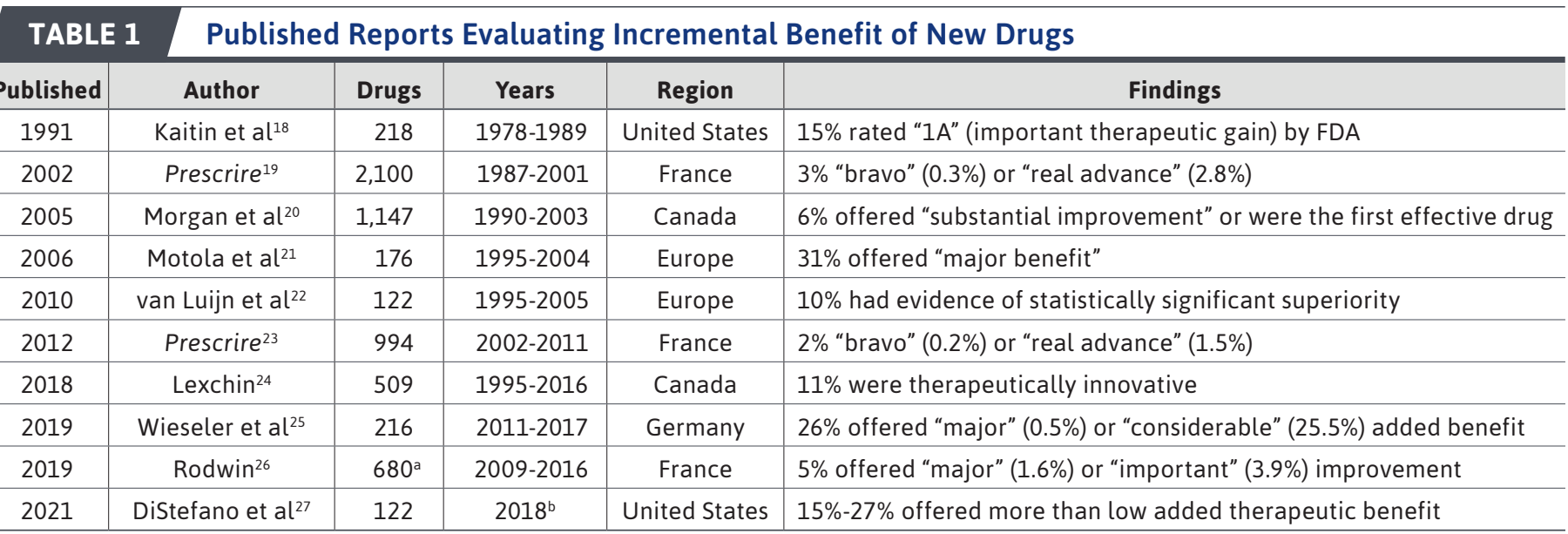

aEstimate based on disclosed average of 85.7 drugs per year.

"DiStefano et al identified 122 "ultra-expensive" drugs based on Medicare Part D spending in 2018.

$F D A=U S$ Food and Drug Administration.

efficacy that the law actually requires. For example, accelerated approval (which relies on surrogate endpoints that are reasonably likely to predict clinical outcomes) requires "meaningful therapeutic benefit...over existing treatments," but the 1992 regulations did not define "meaningful."

Eteplirsen (Exondis 51) was approved under this program in 2016 for the treatment of Duchenne muscular dystrophy over the objections of the FDA review team that there was "no clear evidence of efficacy." ${ }^{5}$ More generally, the FDA has acknowledged that surrogate endpoints, which are the touchstone of the accelerated approval program, "may not in fact be causally related" to clinical endpoints and that, even if causally related, "the drug may have a smaller than expected benefit," since even perfect correlation does not imply any particular magnitude of benefit. ${ }^{6}$

Similarly, the 2012 breakthrough therapy program included the criterion of "substantial improvement over existing therapies," but this criterion could be met even if a new drug was no more effective than available alternatives (eg, if it offered a safety advantage). ${ }^{7}$ Among 58 oncology drugs approved from 2012 to 2017, there was no statistically significant difference in median solid tumor response rates between drugs designated as breakthrough and nonbreakthrough. ${ }^{8}$ Among the 16 breakthrough-designated oncology treatments approved from 2014 to 2016, a median of 57\% of patients experienced no benefit per prespecified criteria, and for 13 (81\%) of these drugs, $8.5 \%$ of patients or fewer experienced complete responses. ${ }^{9}$ Pimavanserin (Nuplazid), a drug used to treat Parkinson disease psychosis, was designated as a breakthrough therapy and approved despite failing to show efficacy in 2 double-blind, placebo-controlled trials. ${ }^{8}$ Approval then occurred following a third trial that shifted the assessment to a purpose-built scale, which showed a 3-point benefit out of 45 possible points.

Expedited programs have been applied to the approval of a few highly effective drugs, such as imatinib (Gleevec, 2001) for cancer (Orphan Drug Act, fast-track, accelerated approval), and sofosbuvir (Sovaldi, 2013) for hepatitis C virus (fast-track, breakthrough therapy). But the fact that the literature continues to reference examples of transformative medicines approved 8 or 20 years ago (of approximately 654 new molecular entities approved from 2001 to 2020) suggests the rarity of true breakthroughs, consistent with published assessments (Table 1).

Even when drugs provide large benefits, perceptions of efficacy can generously outpace the evidence. An 83\% remission rate was widely reported for tisagenlecleucel (Kymriah), a CAR-T therapy approved in 2017 for leukemia (Orphan Drug Act, breakthrough therapy), but this figure was based on assessments within 3 months after treatment and did not include subjects who later relapsed or died during a median follow-up of just 4.8 months. Far fewer $(46 \%)$ were in remission and not censored by the study's end. ${ }^{10}$ Voretigene neparvovec-xioi (Luxturna), a gene therapy that was approved in 2017 to treat a rare inherited blindness (Orphan Drug Act, breakthrough therapy), was widely reported to be curative even though the evidence did not support this claim. ${ }^{11}$ Nusinersen (Spinraza), a costly treatment for a rare muscle-wasting disease (Orphan Drug Act, fast-track), was hailed as "dramatically effective" even 
though most (59\%) subjects in its pivotal trial did not respond according to prespecified criteria. $^{12}$

Expedited programs are most defensible when applied to drugs that offer large incremental benefits. However, the average share of drugs qualifying for at least 1 expedited program (excluding priority review) was $59 \%$ from 2010 to $2019,{ }^{13}$ far exceeding the share of drugs offering major therapeutic gains (Table 1). Of 135 drug-indication pairs approved from 1999 to 2012 for which qualityadjusted life-year (QALY) data were available, 46 (34\%) provided a median incremental benefit of about 0.1 QALY, and another 59 (44\%) offered a median gain of just 0.003 QALY. ${ }^{14}$

Early in clinical development it can be difficult to predict which drugs will be beneficial, and it may be appropriate for regulators to shift additional resources to promising early-stage drugs at the risk of later disappointment. To its credit, the FDA has on average applied expedited programs to those drugs later determined to be more beneficial, as measured by QALYs. ${ }^{13}$ However, even among the 7\% $(10 / 135)$ of drug-indication pairs that received 3 simultaneous program designations, median benefits were less than 0.4 QALY. Certain biases could make even these modest benefits appear larger than they actually are. For example, assumptions about the correlation between surrogate and clinical endpoints that underlie QALY calculations may be false; publication bias can mean studies with QALY gains are more likely to be reported; and studies where QALY gains are unlikely may not be undertaken at all.

The modest benefits offered by most drugs receiving expedited treatment raise questions about the wisdom of reducing evidence requirements to expedite their availability. It is true that patients with life-threatening diseases may be willing to risk the absence of meaningful benefit and possibility of harm. But optimism bias may lead patients to substantially overestimate the magnitude of incremental benefit that a new drug is likely to provide. The interests of future patients are also important, and the failure to adequately test new treatments before approval can delay data collection ${ }^{15}$ and deprive those patients of a fully informed treatment decision. Inadequate evidence that obscures limited efficacy can also lead to wasted resources (see Ferries et al, "FDA Expedited Approval and Implications for Rational Formulary and Health Plan Design, in this issue), create false hope, reduce motivation to undertake preventive efforts, and divert patients from alternate therapeutic options mistakenly believed to be less effective.

Even when a new drug provides large benefits, it is not clear that earlier approval is necessary to help patients with immediate needs. Since 1987, the FDA has administered an expanded access (or "compassionate use") program that allows patients to request experimental therapies before approval, and the FDA nearly always approves these requests. ${ }^{16}$ Although the program depends on the ability and willingness of manufacturers to provide their products and access is not guaranteed, broadening expanded access for today's patients is an alternative to impairing evidence collection in a way that may adversely impact all future patients.

Expedited programs took root during the 1980s when the AIDS crisis presented a new and deadly infectious disease threat with no effective treatments, representing an uncommon circumstance for which an expedited approach may have been particularly suited. The SARS-CoV-2 pandemic presented a similar scenario of a new infectious disease for which no treatments or vaccines were available, although earlier efforts to address SARS-CoV-1 and Ebola meant that research on products such as remdesivir (Veklury) were already underway when a new need suddenly arose.

Historically, however, few noninfectious disease categories have been as amenable to preventive, curative, or near-curative treatment as newly emerging infectious diseases, making the case for expedited approval less compelling in other therapeutic areas. For most drugs, policymakers should reevaluate whether the pendulum has swung too far, leading to more rapid approval of costly medicines that some believe are more prone to adverse events $^{17}$ and that-most importantlypast experience suggests are unlikely to substantially improve or extend patients' lives.

\section{DISCLOSURES}

This commentary is based on work by the author that was supported by Arnold Ventures and the Harvard-MIT Center for Regulatory Science. The funders had no role in the writing of this commentary, or the decision to submit for publication. The author has nothing else to disclose.

\section{ACKNOWLEDGMENTS}

The author gratefully acknowledges Aaron Kesselheim, Michael Rupp, Stephen Darrow, and Domenico Motola for offering input.

\section{REFERENCES}

1. Darrow JJ, Avorn J, Kesselheim AS. Speed, safety, and industry funding: from PDUFA I to PDUFA VI. N Engl J Med. 2017;377(23):2278-86.

2. Darrow JJ. Pharmaceutical efficacy: the illusory legal standard. Wash. E Lee L. Rev. 2013;70:2073-136.

3. HR Rep No. 97-840(I) (1982), reprinted in 1982 U.S.C.C.A.N. 3577. 
4. Investigational new drug, antibiotic, and biological drug product regulations; procedures for drugs intended to treat life-threatening and severely debilitating illnesses. Fed Regist. 1988;53(204):41516-24.

5. US Food and Drug Administration. Medical review (eteplirsen, 206488Orig1s000). July 20, 2016. Accessed April 13, 2021. https://www. accessdata.fda.gov/drugsatfda docs/ nda/2016/206488orig1s000medr.pdf

6. US Food and Drug Administration. New drug, antibiotic, and biological drug product regulations; accelerated approval. Fed Regist. 1992;57(239):58942-60.

7. US Food and Drug Administration. Guidance for industry: expedited programs for serious conditions-drugs and biologics. May 2014. Accessed April 13, 2021. https://www.fda.gov/media/86377/ download

8. Hwang TJ, Franklin JM, Chen CT, et al. Efficacy, safety, and regulatory approval of Food and Drug Administrationdesignated breakthrough and nonbreakthrough cancer medicines. J Clin Onc. 2018;36(18):1805-12.

9. Darrow JJ, Avorn J, Kesselheim AS. The FDA breakthrough drug designation: four years of experience. $\mathrm{N}$ Engl J Med. 2018;378(15):1444-53.

10. Tessema F, Darrow JJ. A new approach to treat childhood leukemia: Novartis' CAR-T therapy. J Law Med Ethics 2017;45:692-97.

11. Darrow JJ. Luxturna: FDA documents reveal the value of a costly gene therapy. Drug Discov Today. 2019;4(2):949-54.
12. Darrow JJ, Sharma M, Shroff M, Wagner AK. Efficacy and costs of spinal muscular atrophy drugs. Sci Trans Med. 2020;12(659):eaay9648.

13. Darrow JJ, Avorn JA, Kesselheim AS. FDA regulation and approval of pharmaceuticals, 1983-2018. JAMA 2020;323(2):164-76.

14. Chambers JD, Thorat T, Wilkinson CL, Neumann PJ. Drugs cleared through the FDA's expedited review offer greater gains than drugs approved by conventional process. Health Aff (Millwood). 2017;8:1408-15.

15. Woloshin S, Schwartz LM, White B, Moore TJ. The fate of FDA postapproval studies. N Engl J Med 377(12):1114-17.

16. Darrow JJ, Sarpatwari A, Avorn J, Kesselheim AS. Practical, legal, and ethical issues in expanded access to investigational drugs. N Engl J Med 2015;372(3):279-86.

17. Darrow JJ, Avorn J, Kesselheim AS. New FDA breakthrough-drug category: implications for patients. N Engl J Med 2014;370(13):1252-58.

18. Kaitin KI, Phelan NR, Raiford D, Morris B. Therapeutic ratings and end-of-phase II conferences: initiatives to accelerate the availability of important new drugs. J Clin Pharmacol 1991;31(1):17-24.

19. Drugs in 2001: a number of ruses unveiled. Prescrire Int. 2002;11(58):58-60.

20. Morgan SG, Bassett KL, Wright JM, et al. "Breakthrough" drugs and growth in expenditure on prescription drugs in Canada. BMJ 2005;331(7520):815-16.
21. Motola D, De Ponte F, Poluzzi E, et al. An update on the first decade of the European centralized procedure: how many innovative drugs? $\mathrm{Br}$ J Clin Pharmacol 2006;62(5):610-16.

22. van Luijn JCF, Gribnau FWJ, Leufkens HGM, et al. Superior efficacy of new medicines? Eur J Clin Pharmacol 2010;66(5):445-48.

23. New drugs and indications in 2011: France is better focused on patients interests after the Mediator scandal, but stagnation elsewhere. Prescrire Int. 2012;21(126):106-10.

24. Lexchin J. Health Canada's use of expedited review pathways and therapeutic innovation 1995-2016: cross-sectional analysis. BMJ Open 2018;8:e023605.

25. Wieseler B, McGauran N, Kaiser T. New drugs: where did we go wrong and what can we do better? BMJ 2019;366:14340.

26. Rodwin M. What can the US learn from pharmaceutical spending controls in France? Commonwealth Fund Issue Brief. January 11, 2019. April 13, 2021. https://www.commonwealthfund.org/ publications/issue-briefs/2019/nov/ what-can-united-states-learn-drugspending-controls-france

27. DiStefano MJ, Kang S, Yehia F, Morales C, Anderson G. Assessing the added therapeutic benefit of ultra-expensive drugs. Value Health. 2021;24(3):397-403. 